\title{
Pattern transitions in a compressible floating elastic sheet
}

\author{
Oz Oshri* \\ Raymond \&5 Beverly Sackler School of Physics \& Astronomy, Tel Aviv University, Tel Aviv 6997801, Israel \\ Haim Diamant $\ddagger$ \\ Raymond 86 Beverly Sackler School of Chemistry, Tel Aviv University, Tel Aviv 6997801, Israel
}

(Dated: May 16, 2017)

\begin{abstract}
Thin rigid sheets floating on a liquid substrate appear, for example, in coatings and surfactant monolayers. Upon uniaxial compression the sheet undergoes transitions from a compressed flat state to a periodic wrinkled pattern to a localized folded pattern. The stability of these states is determined by the in-plane elasticity of the sheet, its bending rigidity, and the hydrostatics of the underlying liquid. Wrinkles and folds, and the wrinkle-to-fold transition, were previously studied for incompressible sheets. In the present work we extend the theory to include finite compressibility. We analyze the details of the flat-to-wrinkle transition, the effects of compressibility on wrinkling and folding, and the compression field associated with the pattern formation. The state diagram of the floating sheet including all three states is presented.
\end{abstract}

\section{INTRODUCTION}

A thin rigid layer covering a liquid is quite an abundant system. Milk skin and dense Langmuir monolayers [1] are two examples. When a thin sheet is laterally compressed, it buckles. Unlike the Euler buckling [2] of a sheet of paper, which makes a single hill or valley, a sheet that covers a soft substrate buckles in more elaborate shapes. The competition between the sheet's resistance to bend, favoring a single large buckle, and the substrate's resistance to deform, preferring many small wiggles, creates patterns with a characteristic intermediate wavelength [3-5]. The simplest pattern, of periodic uniaxial waves, wrinkles, is typically the first to appear upon lateral compression or confinement [4-7, 9-13]. In this case the deformation is evenly spread across the sheet. In another pattern, which often develops upon further compression of a wrinkled sheet, the deformation gets localized in a finite region, a fold [14-29]. The localization of wrinkles into a fold is intrinsic, i.e., it is not tied to any inhomogeneity in the sheet. The generic patterns of wrinkles and folds are observed in a large variety of systems [11,30], over a wide range of length scales - from single-molecule surfactant layers [1,31-33], to 6-nm-thick nanoparticle layers [16, 18], to polymer sheets with thicknesses of order $10 \mathrm{~nm}[8]$ and $1 \mu \mathrm{m}[16,19]$.

The case of a liquid substrate, whose resistance to deformation arises from its weight, has attracted particular attention. The fact that the hydrostatic force on the sheet acts only normal to it, makes this highly nonlinear problem more tractable than similar ones in thin-sheet elasticity. For example, the problem of an infinite, incompressible, floating sheet is exactly solvable $[22,24,25]$, and so is the nonlinear wrinkling of a finite incompressible sheet [29]. The theory reveals a second-order wrinkle-to-fold transition, and a narrow stability region for wrinkles, which disappears in the limit of an infinite sheet. Thus, wrinkles in floating sheets turn out to be a finite-size phenomenon [20]. The theory was found to fit the experiments to a remarkable accuracy with no fitting parameters [23]. (We note that patterns in floating thin sheets can originate from other effects such as surface tension [8,9,34-38]; the present work is restricted to substrate effects that are purely hydrostatic.)

Up until now the theory of floating sheets has been limited to incompressible layers. Indeed, as the sheet is made increasingly thin, its resistance to bending becomes increasingly weaker than its resistance to compression - while the compression modulus depends linearly on the thickness $t$, the bending modulus scales as $t^{3}$ [2]. Hence, the assumption of incompressibility is valid for a sufficiently small thickness. It does not allow, however, to examine in detail the onset of wrinkling, i.e., the transition from a compressed flat configuration to a wrinkled one; in the absence of compressibility, wrinkles must appear at an arbitrarily small confinement. In addition, it is of interest to study the evolution of the compression field accompanying the pattern formation, as well as the corrections to earlier results due to compressibility. These are the purposes of the present work.

We begin in Sec. II by introducing the model and its resulting energy functional. Section III presents a detailed analysis of the energy-minimizing configurations. Following the footsteps of Refs. [21, 29], we employ a multiple-scale

\footnotetext{
* ozzoshri@tau.ac.il

$\dagger$ Present address: Department of Chemical and Petroleum Engineering, University of Pittsburgh, PA 15261, USA

‡ hdiamant@tau.ac.il
} 
analysis to study the flat-to-wrinkle transition, wrinkle growth, the wrinkle-to-fold transition, and the subsequent evolution of the fold. In Sec. IV we conclude and discuss the experimental relevance of the findings.

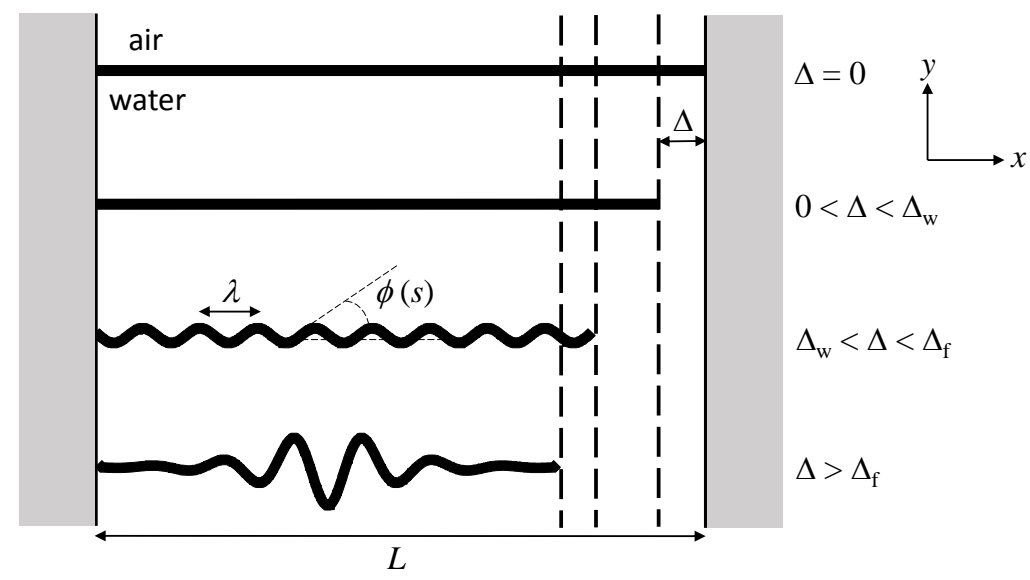

FIG. 1. Schematic evolution of patterns in a compressible finite sheet floating on a heavy liquid. For small displacements, $\Delta<\Delta_{\mathrm{w}}$, the sheet is compressed while remaining flat. At a critical displacement $\Delta_{\mathrm{w}}$ the flat sheet buckles into wrinkles of wavelength $\lambda$. For $\Delta_{\mathrm{w}}<\Delta<\Delta_{\mathrm{f}}$ the wrinkles grow in amplitude until, at another critical displacement $\Delta_{\mathrm{f}}$, the pattern begins to localize. For $\Delta>\Delta_{\mathrm{f}}$ the pattern continues to localize into a single fold.

\section{MODEL}

A compressible thin elastic sheet of relaxed length $L$, bending modulus $B$, and stretching modulus $Y$, lies on a fluid substrate of mass density $\rho$. The sheet is uniaxially compressed from the boundaries and develops an out-of-plane deformation on the $x y$ plane, independent of $z$ (see Fig. 1). This configuration makes the problem one-dimensional, dependent solely on the arclength along the sheet. We distinguish between the relaxed arclength, $s$, and the compressed one, $\hat{s}$. The deformation of the sheet is defined by two functions: a compression field, $\gamma(s)=d \hat{s} / d s[(\gamma-1)$ being the in-plane strain], and the angle profile $\phi(s)$ between the tangent to the sheet and the horizontal axis. (The local curvature is then given by $v \equiv d \phi / d \hat{s}=\gamma^{-1} d \phi / d s$.) It is convenient to define also the height function $h(s)$, which is related to $\gamma$ and $\phi$ by the geometric constraint,

$$
\frac{d h}{d \hat{s}}=\gamma^{-1} \frac{d h}{d s}=\sin \phi .
$$

The energy (per unit length along $z$ ) of a given deformation contains contributions from stretching, bending, and the fluid's hydrostatics,

$$
\begin{aligned}
E & =E_{\mathrm{s}}+E_{\mathrm{b}}+E_{\mathrm{f}}, \\
E_{\mathrm{s}} & =\int_{-L / 2}^{L / 2} d s \frac{Y}{2}(\gamma-1)^{2}, \\
E_{\mathrm{b}} & =\int_{-L / 2}^{L / 2} d s \frac{B}{2}\left(\frac{d \phi}{d s}\right)^{2}, \\
E_{\mathrm{f}} & =\int_{-L / 2}^{L / 2} d s \frac{\rho g}{2} h^{2} \cos \phi .
\end{aligned}
$$

The boundary displacement associated with a given deformation is

$$
\Delta=\int_{-L / 2}^{L / 2} d s(1-\gamma \cos \phi) .
$$


We note that $E_{\mathrm{s}}+E_{\mathrm{b}}$ as given in Eq. (2) is the known energy functional of the extensible elastica [40, 41]. It can be systematically derived from the continuous limit of a discrete model, which ensures the independence of the stretching and bending contributions [41]. The equilibrium deformation of the sheet is the minimizer of $E$ for a given $\Delta$, subject to the geometrical constraint (1). Alternatively, instead of $\Delta$, we can specify the boundary pressure (force per unit length) $P$ and minimize

$$
G=E-P \Delta
$$

for a given $P$.

The problem has two intrinsic lengths (apart from the system length $L$ ),

$$
\begin{aligned}
\xi & \equiv(B / Y)^{1 / 2}, \\
q^{-1} & =\lambda /(2 \pi) \equiv[B /(\rho g)]^{1 / 4},
\end{aligned}
$$

which define a dimensionless compressibility parameter,

$$
\zeta \equiv(q \xi)^{2}=(B \rho g)^{1 / 2} / Y .
$$

For an incompressible sheet, $Y \rightarrow \infty$, and so $\zeta \rightarrow 0$. For a compressible sheet, in terms of the sheet thickness $t$, we have $B \sim t^{3}$ and $Y \sim t$, implying $\xi \sim t, q^{-1} \sim t^{3 / 4}$, and $\zeta \sim t^{1 / 2} \stackrel{t \rightarrow 0}{\longrightarrow} 0$. Thus, $\zeta$ is small for thin sheets. The analysis given in Sec. III yields expressions which are correct to all orders in $\zeta$.

We rescale all lengths by $q^{-1}$, the energies per unit length by $B q$, and the pressure by $\zeta Y=(B \rho g)^{1 / 2}$. This results in

$$
G=\int_{-L / 2}^{L / 2} d s\left[\frac{1}{2 \zeta}(\gamma-1)^{2}+\frac{1}{2}\left(\frac{d \phi}{d s}\right)^{2}+\frac{1}{2} \gamma h^{2} \cos \phi-P(1-\gamma \cos \phi)\right]
$$

Thus, the problem contains three dimensionless parameters: $\zeta$ and the rescaled $P$ and $L$.

\section{DETAILED ANALYSIS}

As in earlier works $[22,24,29]$, we first recast the problem in dynamical terms, where $s$ stands for time and $\left({ }^{*}\right)$ denotes a derivative with respect to $s$. In this analogy we need to minimize an "action", $\mathcal{S}=\int_{-L / 2}^{L / 2} d s \mathcal{L}(\phi, h, \gamma, \dot{\phi}, \dot{h}, \dot{\gamma})$, with the "Lagrangian",

$$
\mathcal{L}=\frac{1}{2} \dot{\phi}^{2}+\frac{1}{2 \zeta}(\gamma-1)^{2}+\frac{1}{2} \gamma h^{2} \cos \phi-P(1-\gamma \cos \phi)-Q(s)(\gamma \sin \phi-\dot{h}) .
$$

In Eq. (9) $Q(s)$ is a Lagrange multiplier introduced to impose the local geometrical constraint (1) at each point along the sheet. The conjugate "momenta" are identified as $p_{\phi}=\partial \mathcal{L} / \partial \dot{\phi}=\dot{\phi}, p_{h}=\partial \mathcal{L} / \partial \dot{h}=Q$, and $p_{\gamma}=\partial \mathcal{L} / \partial \dot{\gamma}=0$. These are used to construct the "Hamiltonian", $\mathcal{H}\left(\phi, h, \gamma, p_{\phi}, p_{h}, p_{\gamma}\right)=p_{\phi} \dot{\phi}+p_{h} \dot{h}+p_{\gamma} \dot{\gamma}-\mathcal{L}$,

$$
\mathcal{H}=-\frac{1}{2 \zeta}(\gamma-1)^{2}+\frac{1}{2} p_{\phi}^{2}-\left(\frac{1}{2} h^{2}+P\right) \gamma \cos \phi+p_{h} \gamma \sin \phi=\mathcal{H}_{0}=\text { const. }
$$

As $\mathcal{L}$ has no explicit dependence on $s, \mathcal{H}=\mathcal{H}_{0}$ is a "constant of motion", i.e., it is uniform across the sheet and fully determined by the conditions at the boundaries.

\section{A. General relation between compression and curvature}

The fact that the energy does not depend on $\dot{\gamma}$ has led above to $p_{\gamma}=0$. This results, through Hamilton's equation, $\dot{p}_{\gamma}=-\partial \mathcal{H} / \partial \gamma=0$, in

$$
\frac{1}{\zeta}(\gamma-1)+\left(\frac{1}{2} h^{2}+P\right) \cos \phi-p_{h} \sin \phi=0
$$


Equations (10) and (11) yield a general relation between the compression and angle fields,

$$
\gamma(s)=\left[\bar{\gamma}^{2}-\zeta\left(\frac{d \phi}{d s}\right)^{2}\right]^{1 / 2},
$$

where $\bar{\gamma}=1+2 \zeta \mathcal{H}_{0}$ is a constant determined by boundary conditions.

This relation raises several noteworthy points. (i) In the incompressible limit $(\zeta=0), \gamma(s) \equiv 1$ as expected. (ii) In flat configurations, the compression field has a uniform value, $\gamma(s) \equiv \bar{\gamma}$, dictated by boundary conditions. As the sheet goes out of plane, the compression is increased throughout the sheet, $\gamma(s)<\bar{\gamma}$, with maxima at the points of maximum curvature. (In the case of uniaxial deformation, unlike the case of a two-dimensional one, buckling does not cause stress collapse.) (iii) If the sheet is hinged at its boundaries (having zero curvature there), $\bar{\gamma}$ is the boundary value of the compression field, and $\gamma(s)$ is smaller than that value everywhere in the sheet. (iv) The relation (12) is independent of the hydrostatic contribution $E_{\mathrm{f}}$; the dependence on $\rho g$ enters through factors of $q^{2}$ in both $\zeta$ and the rescaled $d s^{2}$, which mutually cancel. In fact, the same relation holds for any energy of the form $E_{\mathrm{s}}+E_{\mathrm{b}}+\int d s \gamma V(\phi)$, where $V(\phi)$ is an arbitrary function [41]. (v) In terms of the curvature, $v=d \phi / d \hat{s}=\gamma^{-1} d \phi / d s$, Eq. (12) takes the form,

$$
\gamma(\hat{s})=\frac{\bar{\gamma}}{\sqrt{1+\zeta v^{2}}}
$$

This relation between stretching and curvature is reminiscent of relativistic mechanics, where $v,-\zeta^{-1 / 2}$, and $\gamma$ play the roles, respectively, of velocity, speed of light, and the Lorentz dilation factor, such that the incompressible limit corresponds to classical mechanics. This curious (and useful) analogy is discussed in Ref. [41].

\section{B. States and transitions}

In this section we derive expressions for the in-plane deformation, out-of-plane profile, and critical values of pressure and displacement at the two transitions. The expressions are valid for an arbitrary size of the compressibility parameter $\zeta$. However, since this parameter is typically very small, a leading-order expansion in $\zeta$ should be sufficient for all practical purposes, and we provide these approximate expressions as well.

We begin with the flat state. Specializing Eqs. (3), (8), and (12) to the case of $h(s)=\phi(s) \equiv 0$, we obtain

$$
\begin{aligned}
\gamma(s) & \equiv \bar{\gamma}=1-\Delta / L=1-\zeta P, \\
\Delta & =L \zeta P, \\
G & =-L \zeta P^{2} / 2,
\end{aligned}
$$

which describes the simple linear deformation of a uniaxially compressed flat sheet.

To analyze the non-planar patterns we employ a multiple-scale analysis similar to the one presented in Refs. [21, 29]. We assume a long (but finite) sheet, $L \gg k^{-1}$, where $2 \pi / k \sim \lambda$ is the wrinkle wavelength, and $\lambda$ is the intrinsic length defined in Eq. (6). Our small parameter is the distance to the flat-to-wrinkle transition; more specifically,

$$
\epsilon \equiv\left(P_{\mathrm{w}}-P\right)^{1 / 2} .
$$

As $L$ is increased to arbitrarily large values, the wrinkle-to-fold transition occurs arbitrarily close to the flat-to-wrinkle one $[20,21]$. Thus, restricting the analysis to the leading order in $1 / L$ allows the use of the same perturbation theory for both transitions.

For simplicity, we limit the discussion to $L=\pi N / k$, where $N$ is an integer, i.e., the wrinkled pattern contains $N$ wrinkles. Odd and even $N$ correspond respectively to symmetric and antisymmetric solutions. In addition, we assume hinged boundary conditions, i.e., vanishing height and bending moment at the edges of the sheet,

$$
h( \pm L / 2)=\dot{\phi}( \pm L / 2)=0 .
$$

For these boundary conditions, according to Eq. (12), the constant $\bar{\gamma}$ coincides with the boundary compression $\gamma( \pm L / 2)$.

In the wrinkled state the pattern is periodic, and slightly above the wrinkle-to-fold transition it is weakly localized. Thus, the system exhibits either one spatial variation, or two with well-separated scales - fast undulations of wavelength $2 \pi / k \sim \lambda$, and a slow envelope of decay length $\eta^{-1} \gg \lambda$. This suggests the following approximation:

$$
h(s) \simeq \epsilon \cos (k s) H(S),
$$


where $S \equiv \epsilon s$ is the slow variable, and we will denote a derivative with respect to $S$ by ()$^{\prime}$. In the wrinkled state $H$ is constant, and in the folded state it is a decaying envelope. We have chosen a symmetric profile; an antisymmetric one is obtained by replacing the cosine with a sine and leads to similar results. The boundary conditions of vanishing height are satisfied by the fast oscillating function, $\cos (N \pi / 2)=0$ for odd $N$. The boundary conditions of vanishing bending moment turn into

$$
H^{\prime}( \pm \epsilon L / 2)=0
$$

We substitute the height function (17) in the equations derived above (specifically, Eqs. (1), (3), (8), and (12)), average over the fast oscillations, and obtain approximate expressions for the various quantities,

$$
\begin{aligned}
G & \simeq G_{\mathrm{w}}+\epsilon^{2} G_{2}+\epsilon^{4} G_{4}, & & G_{\mathrm{w}}=-\zeta P_{\mathrm{w}} / 2, \\
\Delta & \simeq \Delta_{\mathrm{w}}+\epsilon^{2} \Delta_{2}+\epsilon^{4} \Delta_{4}, & & \Delta_{\mathrm{w}}=L \zeta P_{\mathrm{w}}, \\
\bar{\gamma} & \simeq \bar{\gamma}_{\mathrm{w}}+\epsilon^{2} \bar{\gamma}_{2}+\epsilon^{4} \bar{\gamma}_{4}, & & \bar{\gamma}_{\mathrm{w}}=1-\zeta P_{\mathrm{w}}, \\
\gamma(s) & \simeq \gamma_{\mathrm{w}}+\epsilon^{2} \gamma_{2}(s)+\epsilon^{4} \gamma_{4}(s), & & \gamma_{\mathrm{w}}=\bar{\gamma}_{\mathrm{w}}=1-\zeta P_{\mathrm{w}},
\end{aligned}
$$

where the zeroth-order terms, the ones at the wrinkle-to-fold transition, have been obtained from the edge of the flat state, Eq. (14). Since the system has an up-down symmetry, only even powers of $\epsilon$ survive. The assumed height function (17), linear in $\epsilon$, is sufficient for getting the leading terms specified in Eq. (19). The expansion is worked out in detail in the Supplementary Material [42].

\section{Flat-to-wrinkle transition and wrinkle growth}

The expression for the second-order energy term, $G_{2}$, is found to be [42]

$$
G_{2}=\left(1-\gamma_{\mathrm{w}}\right) L+\frac{1}{4 \gamma_{\mathrm{w}}^{2}}\left(k^{4}-\gamma_{\mathrm{w}} P_{\mathrm{w}} k^{2}+\gamma_{\mathrm{w}}^{3}\right) \int_{-L / 2}^{L / 2} H^{2} d s .
$$

The flat state becomes unstable when going out of plane lowers the energy, i.e., when the coefficient of $\int H^{2}$ in $G_{2}$ becomes negative, which is obtained when the pressure exceeds $P_{\mathrm{w}}=\left(k^{4}+\gamma_{\mathrm{w}}^{3}\right) /\left(k^{2} \gamma_{\mathrm{w}}\right)$. This occurs first for a critical wavenumber, $k=k_{\mathrm{c}}=\gamma_{\mathrm{w}}^{3 / 4}$, whereby $P_{\mathrm{w}}=2 \gamma_{\mathrm{w}}^{1 / 2}$. Adding to these results the known conditions at the edge of the flat state, Eq. (19), and solving for $P_{\mathrm{w}}$, we obtain the critical flat-to-wrinkle wavenumber, pressure, displacement, and compression as functions of the compressibility parameter alone,

$$
\begin{aligned}
k_{\mathrm{c}}(\zeta) & =\left(\sqrt{1+\zeta^{2}}-\zeta\right)^{3 / 2} \simeq 1-3 \zeta / 2, \\
P_{\mathrm{w}}(\zeta) & =2\left(\sqrt{1+\zeta^{2}}-\zeta\right) \simeq 2(1-\zeta), \\
\Delta_{\mathrm{w}}(\zeta) & =2 L \zeta\left(\sqrt{1+\zeta^{2}}-\zeta\right) \simeq 2 L \zeta . \\
\gamma_{\mathrm{w}}(\zeta) & =1-2 \zeta\left(\sqrt{1+\zeta^{2}}-\zeta\right) \simeq 1-2 \zeta .
\end{aligned}
$$

Equation (21) extends the results for the wrinkling transition in an incompressible sheet $[4]\left(k_{\mathrm{c}}=1, P_{\mathrm{w}}=2, \Delta_{\mathrm{w}}=0\right.$, $\left.\gamma_{\mathrm{w}}=1\right)$ to sheets of finite compressibility. We find that the compressibility lowers the critical pressure and increases the wrinkle wavelength. In addition, we obtain the second-order expressions for the displacement and compression $[42]$,

$$
\begin{aligned}
\Delta_{2} & =\frac{1}{4} \sqrt{1+\zeta^{2}} \int_{L / 2}^{L / 2} H^{2} d s-L \zeta, \\
\gamma_{2}(s) & =\zeta\left[1-\frac{1}{2} \cos ^{2}\left(k_{\mathrm{c}} s\right) H^{2}(S)\right] .
\end{aligned}
$$

Thus, as confinement is increased and wrinkles grow, both the mean compression and its undulations increase as well.

To get the details of the wrinkled state beyond the transition, we need the height profile that minimizes the energy. This requires the fourth-order energy term [42],

$$
G_{4}=-L \zeta / 2+\frac{2}{k_{\mathrm{c}}^{2 / 3}} \int_{-L / 2}^{L / 2}\left[\frac{1}{2}\left(H^{\prime}\right)^{2}-\frac{\alpha}{4} H^{4}+\frac{\beta}{2} H^{2}-\frac{1}{4}\left(H H^{\prime}\right)^{\prime}\right] d s,
$$


where

$$
\alpha=\frac{1}{8}\left[1-\frac{5}{4} \zeta\left(\sqrt{1+\zeta^{2}}-\zeta\right)\right], \quad \beta=\frac{1}{4}\left[1-\zeta\left(\sqrt{1+\zeta^{2}}-\zeta\right)\right]
$$

are strictly positive functions of $\zeta$. The last (total-derivative) term in Eq. (24) vanishes due to the hinged boundary conditions (18). Minimizing $G_{4}$ with respect to $H(S)$ yields the following amplitude equation:

$$
H^{\prime \prime}+\alpha H^{3}-\beta H=0 .
$$

Equation (26) is to be solved together with the boundary conditions (18).

One solution is a constant,

$$
H(S) \equiv H_{0}(\zeta)=\sqrt{\beta / \alpha} \simeq \sqrt{2}(1+\zeta / 8) .
$$

This corresponds to periodic wrinkles,

$$
h(s)=A \cos \left(k_{\mathrm{c}} s\right), \quad A=\epsilon H_{0},
$$

whose amplitude grows with the drop in pressure as

$$
A(P)=H_{0}(\zeta)\left(P_{\mathrm{w}}-P\right)^{1 / 2} \simeq \sqrt{2}\left(P_{\mathrm{w}}-P\right)^{1 / 2}(1+\zeta / 8) .
$$

To get the amplitude as a function of displacement we substitute $H=H_{0}$ in Eq. (22), finding

$$
A(\Delta)=2\left(\frac{\Delta-\Delta_{\mathrm{w}}}{L}\right)^{1 / 2}\left(\sqrt{1+\zeta^{2}}-4 \zeta / H_{0}^{2}\right)^{-1 / 2} \simeq 2\left(\frac{\Delta-\Delta_{\mathrm{w}}}{L}\right)^{1 / 2}(1+\zeta) .
$$

This, together with Eq. (15), yields the pressure-displacement relation,

$$
P(\Delta)=P_{\mathrm{w}}-\frac{4\left(\Delta-\Delta_{\mathrm{w}}\right)}{L}\left(H_{0}^{2} \sqrt{1+\zeta^{2}}-4 \zeta\right)^{-1} \simeq P_{\mathrm{w}}-2 \frac{\Delta-\Delta_{\mathrm{w}}}{L}(1+7 \zeta / 4) .
$$

Equations (27)-(31) generalize the results known for wrinkles in incompressible sheets [20-22] $\left(H_{0}=\sqrt{2}, A=\right.$ $\left.\sqrt{2\left(P_{\mathrm{w}}-P\right)}=2 \sqrt{\Delta / L}, P=P_{\mathrm{w}}-2 \Delta / L\right)$ to compressible sheets. Note that the seeming increase of amplitude with compressibility in Eqs. (29) and (30) is misleading; once the dependence of $P_{\mathrm{w}}$ and $\Delta_{\mathrm{w}}$ on $\zeta$ is included, the amplitude at fixed $P$ or $\Delta$ decreases with $\zeta$, as intuitively expected.

We also calculate the compression field along the sheet by substituting the wrinkled profile (28) in Eq. (23),

$$
\gamma(s)=\gamma_{\mathrm{w}}+\zeta\left(P_{\mathrm{w}}-P\right)\left[1-\frac{1}{2} H_{0}^{2} \cos ^{2}\left(k_{\mathrm{c}} s\right)\right] \simeq \gamma_{\mathrm{w}}+\zeta\left(P_{\mathrm{w}}-P\right)\left[1-\cos ^{2}\left(k_{\mathrm{c}} s\right)\right]
$$

or, in terms of displacement,

$$
\gamma(s)=\gamma_{\mathrm{w}}+\frac{4 \zeta\left(\Delta-\Delta_{\mathrm{w}}\right)}{L}\left(H_{0}^{2} \sqrt{1+\zeta^{2}}-4 \zeta\right)^{-1}\left(1-\frac{1}{2} H_{0}^{2} \cos ^{2}\left(k_{\mathrm{c}} s\right)\right) \simeq \gamma_{\mathrm{w}}+2 \zeta \frac{\Delta-\Delta_{\mathrm{w}}}{L}\left[1-\cos ^{2}\left(k_{\mathrm{c}} s\right)\right] .
$$

The undulating compression field has double the frequency of the wrinkles. Around the wrinkles' extrema (both minima and maxima) there is some extra compression, which takes some of the imposed displacement and makes the wrinkle amplitude slightly smaller than it would have been in the incompressible case.

The wrinkle amplitude $A$ can be defined as the order parameter of the flat-to-wrinkle transition. Equations (29) and (30) show that the transition is second-order, as in the incompressible case.

Figure 2(a) shows the wrinkled profile of the compressible sheet along with its incompressible counterpart (dotted curve). One can notice the slightly shorter wavelength in the incompressible case. In Fig. 2(b) we see the corresponding compression field (minus its uniform value at the wrinkling threshold). 

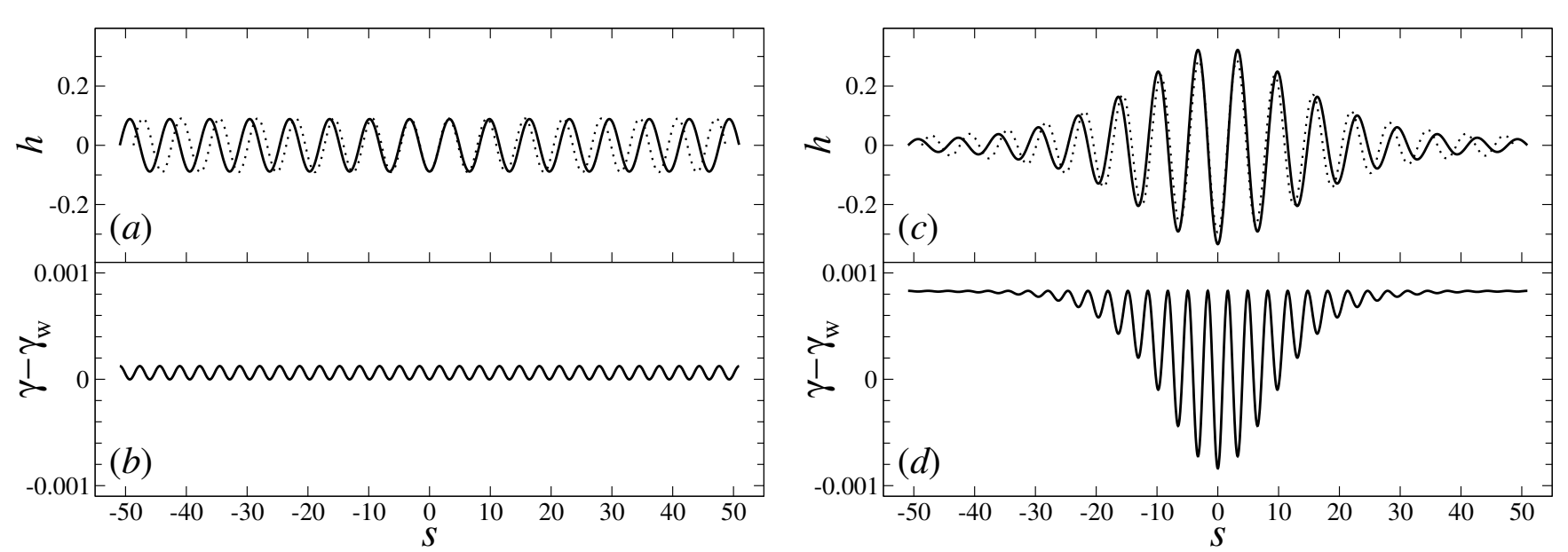

FIG. 2. Height $(\mathrm{a}, \mathrm{c})$ and compression (b,d) profiles, as a function of arclength, in the wrinkled state (a,b) and folded state $(c, d)$. The height profiles of the corresponding incompressible sheet $(\zeta=0$; dotted curves) are shown for comparison. The height $h$ and arclength $s$ are rescaled by the intrinsic length $q^{-1}$ ( $q$ being the wrinkle wavenumber in the incompressible sheet). The compression at the flat-to-wrinkle transition, $\gamma_{\mathrm{w}}$, has been subtracted from the compression field. The selected parameters are $\zeta=0.03$ and $L=31 \pi / k_{\mathrm{c}}$, allowing for a symmetric profile with exactly 16 wrinkles. These yield $\gamma_{\mathrm{w}} \simeq 0.94$ and critical displacements (rescaled by $\left.q^{-1}\right) \Delta_{\mathrm{w}} \simeq 5.93, \Delta_{\mathrm{f}} \simeq 6.32$. The results are for $\Delta=\Delta_{\mathrm{w}}+0.2 \in\left(\Delta_{\mathrm{w}}, \Delta_{\mathrm{f}}\right)(\mathrm{a}, \mathrm{b})$, and $\Delta=\Delta_{\mathrm{w}}+0.6>\Delta_{\mathrm{f}}(\mathrm{c}, \mathrm{d})$. The corresponding values for the incompressible sheet (dotted curves) are $\Delta_{\mathrm{w}}=0, \Delta_{\mathrm{f}} \simeq 0.405$, and $\Delta=0.2$ (a), $\Delta=0.6$ (c), to ensure a valid comparison.

\section{Wrinkle-to-fold transition and fold evolution}

Equations (26) and (18) have other solutions in the form of Jacobi elliptic functions [39]. Out of those twelve functions, only one, dn, is found to provide a physical solution [29],

$$
\begin{aligned}
& H(S)=\sqrt{\frac{2}{\alpha}} \kappa \operatorname{dn}(\kappa S, m), \quad \kappa(m)=\sqrt{\frac{\beta}{2-m}}, \\
& K(m) / \kappa(m)=\epsilon L / 2,
\end{aligned}
$$

where $K(m)$ is the complete elliptic integral of the first kind, which is half the period of the function dn [39]. The resulting height profile is

$$
h(s)=\epsilon \sqrt{\frac{2}{\alpha}} \kappa \cos \left(k_{\mathrm{c}} s\right) \operatorname{dn}(\kappa \epsilon s, m)=\sqrt{\frac{8}{\alpha}} \frac{K(m)}{L} \cos \left(k_{\mathrm{c}} s\right) \operatorname{dn}\left(\frac{2 K(m)}{L} s, m\right) .
$$

For given $P, L$, and $\zeta$, one first solves Eq. (35) to find $m$ and then uses it in Eq. (36) to obtain the profile.

The modulus $m$ takes values in the range $0 \leq m \leq 1$. In the limit $m \rightarrow 0$, the function $\operatorname{dn}(u, m) \rightarrow 1$, leading to

$$
m \rightarrow 0: \quad h(s)=\epsilon \sqrt{\beta / \alpha} \cos \left(k_{\mathrm{c}} s\right),
$$

which coincides with the wrinkled profile obtained above, Eq. (28). For any $m>0$ the function $\operatorname{dn}(u, m)$ is a nonuniform envelope, peaked at $u=0$ and decaying symmetrically on both sides. This breaks the profile's periodicity and localizes it around the origin. Thus, we can define $m$ as the order parameter of the wrinkle-to-fold transition. In the limit $m \rightarrow 1$ we have $\operatorname{dn}(u, m) \rightarrow 1 / \cosh (u)$, which gives the following localized profile:

$$
m \rightarrow 1: \quad h(s)=\epsilon \sqrt{\frac{2 \beta}{\alpha}} \frac{\cos \left(k_{\mathrm{c}} s\right)}{\cosh (\sqrt{\beta} \epsilon s)} .
$$

Next, we express the pressure and displacement as functions of $m$. Equation (35) readily gives

$$
P(m)=P_{\mathrm{w}}-\frac{4}{\beta L^{2}}(2-m) K^{2}(m) \simeq P_{\mathrm{w}}-\frac{16}{L^{2}}(1+\zeta)(2-m) K^{2}(m) .
$$


Within our approximations, the maximum height of the localized profile is proportional to $\epsilon$ even as $m \rightarrow 1$; see Eq. (38). Hence, we may continue to use Eqs. (22) and (23) for the displacement and compression. Substituting in Eq. (22) the envelope $H(S)$ of Eq. (34) and using Eq. (35), we find

$$
\Delta(m)=\Delta_{\mathrm{w}}+\frac{2}{\beta L} K(m)\left[H_{0}^{2} \sqrt{1+\zeta^{2}} E(m)-2 \zeta(2-m) K(m)\right]
$$

where $E(m)$ is the complete elliptic integral of the second kind [39].

To obtain the critical wrinkle-to-fold pressure and displacement we take the limit $m \rightarrow 0$, which yields

$$
\begin{aligned}
& P_{\mathrm{f}}=P_{\mathrm{w}}-\frac{2 \pi^{2}}{L^{2} \beta} \simeq P_{\mathrm{w}}-\frac{8 \pi^{2}}{L^{2}}(1+\zeta), \\
& \Delta_{\mathrm{f}}=\Delta_{\mathrm{w}}+\frac{\pi^{2}}{2 \beta L}\left(H_{0}^{2} \sqrt{1+\zeta^{2}}-2 \zeta\right) \simeq \Delta_{\mathrm{w}}+\frac{4 \pi^{2}}{L}(1+\zeta / 4) .
\end{aligned}
$$

These results show that with increasing $L$ the wrinkle-to-fold transition gets increasingly close to the flat-to-wrinkle one, as stated earlier. Equation (41) extends the results for an incompressible finite sheet $[29]\left(P_{\mathrm{f}}=P_{\mathrm{w}}-8 \pi^{2} / L^{2}\right.$, $\left.\Delta_{\mathrm{f}}=4 \pi^{2} / L\right)$. Compressibility pushes the transition to larger displacement. Expanding $P(m)$ and $\Delta(m)$ in small $m$, we obtain $P_{\mathrm{f}}-P \sim \Delta-\Delta_{\mathrm{f}} \sim m^{2}$. Thus, compressibility does not affect the order of the wrinkle-to-fold transition, which is second-order.

Above the wrinkle-to-fold transition, the pressure-displacement relation is obtained parametrically as $(\Delta(m), P(m))$ from Eqs. (39) and (40). In the localized limit, $m \rightarrow 1$, these equations turn into $P(m) \simeq P_{\mathrm{w}}-\left[4 /\left(\beta L^{2}\right)\right] K^{2}(m)$ and $\Delta(m) \simeq \Delta_{\mathrm{w}}+[2 /(\beta L)] K(m)\left[H_{0}^{2} \sqrt{1+\zeta^{2}}-2 \zeta K(m)\right]$. Eliminating $K(m)$ while keeping only the leading term in $\epsilon$, we find the pressure-displacement relation,

$$
m \rightarrow 1: \quad P(\Delta)=P_{\mathrm{w}}-\frac{\alpha^{2}}{\beta\left(1+\zeta^{2}\right)}\left(\Delta-\Delta_{\mathrm{w}}\right)^{2} \simeq P_{\mathrm{w}}-\frac{1}{16}(1-3 \zeta / 2)\left(\Delta-\Delta_{\mathrm{w}}\right)^{2} .
$$

This localized limit no longer depends on the system size $L$. Equation (42) is to be compared with the result for an infinite incompressible sheet, $P(\Delta)=1-\Delta^{2} / 16$ [22]. We use this result to rewrite the localized profile (38) in terms of the displacement,

$$
\begin{aligned}
m \rightarrow 1: \quad h(s) & =A_{0} \frac{\cos \left(k_{\mathrm{c}} s\right)}{\cosh (\eta s)} \\
A_{0} & =\left(\frac{2 \alpha}{1+\zeta^{2}}\right)^{1 / 2}\left(\Delta-\Delta_{\mathrm{w}}\right) \simeq \frac{\Delta-\Delta_{\mathrm{w}}}{2}(1-5 \zeta / 8), \\
\eta & =\frac{\alpha}{\sqrt{1+\zeta^{2}}}\left(\Delta-\Delta_{\mathrm{w}}\right) \simeq \frac{\Delta-\Delta_{\mathrm{w}}}{8}(1-5 \zeta / 4),
\end{aligned}
$$

which, for $\zeta=0$, coincides with the infinite-length incompressible case $\left(A_{0}=\Delta / 2, \eta=\Delta / 8\right)[22]$. Thus, compressibility is found to lower and widen the fold.

Finally, we substitute $H(S)$ of Eq. (34) in Eq. (23) to obtain the compression field in the folded state,

$$
\gamma(s)=\gamma_{\mathrm{w}}+\epsilon^{2} \zeta\left(1-\frac{\kappa^{2}}{\alpha} \cos ^{2}\left(k_{\mathrm{c}} s\right) \operatorname{dn}^{2}(\kappa \epsilon s, m)\right) \stackrel{m \rightarrow 1}{\longrightarrow} \gamma_{\mathrm{w}}+\frac{1}{2} \zeta A_{0}^{2}\left(\frac{\alpha}{\beta}-\frac{\cos ^{2}\left(k_{\mathrm{c}} s\right)}{\cosh ^{2}(\eta s)}\right)
$$

The maximum compression is at the tip of the fold.

Figure 2(c) presents the height profile of a folded sheet, along with the corresponding profile in the incompressible case (dotted curve). In Fig. 2(d) we show the accompanying localized compression field.

\section{CONCLUSION}

In this work we have analyzed the effect of the compressibility of a finite-size floating sheet on the patterns that the sheet develops upon uniaxial lateral compression. The theory reproduces earlier results for incompressible infinite [22] and finite [29] floating sheets in the appropriate limits. It provides the corrections introduced by finite compressibility. It yields also the nonuniform compression fields (obviously absent in the incompressible case), which accompany the buckling patterns. 

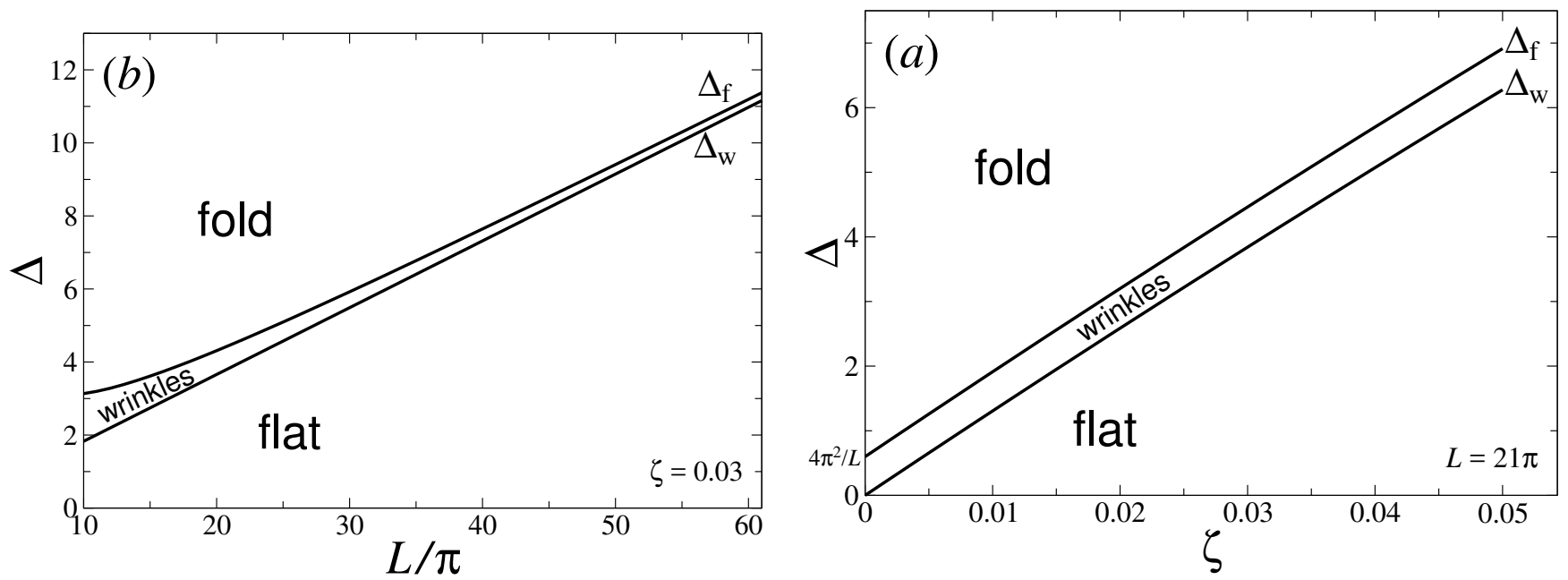

FIG. 3. State diagrams on the $(\Delta, \zeta)$ plane for fixed $L=21 \pi$ (a) and $(\Delta, L)$ plane for fixed $\zeta=0.03$ (b). The displacement $\Delta$ and system length $L$ are rescaled by the intrinsic length $q^{-1}$ ( $q$ being the wrinkle wavenumber of the incompressible sheet).

The obtained information is concisely summarized in the two state diagrams presented in Fig. 3. Figure 3(a) shows the regions of stability of the three states - flat, wrinkled, and folded - on the displacement-compressibility plane for a fixed sheet length. Figure 3(b) shows the stability regions of the same states on the displacement-length plane for a fixed compressibility parameter. The lines separating the stability regions represent second-order transitions. In the limit of an incompressible sheet $(\zeta \rightarrow 0)$ the flat-state region vanishes. The stability region of the wrinkled state is narrow and disappears in the limit of an infinite sheet $(L \rightarrow \infty)$. Thus, diagrams containing all three states require an account of both finite size and finite compressibility, as we have presented here.

By expanding in $\epsilon^{2}=P_{\mathrm{w}}-P$ we have implicitly assumed that $\zeta \gg P_{\mathrm{w}}-P_{\mathrm{f}} \simeq 8 \pi^{2} / L^{2}$. This imposes an upper bound on the sheet thickness $t$ (equivalently, a lower bound on its length $L$ ) for the theory to be valid. In terms of the system's physical parameters the condition reads

$$
t \ll \frac{\rho g L^{2}}{8 \pi^{2} E}
$$

Another practical consideration is that in typical experimental scenarios $\zeta$ is very small. For example, the 10$\mu \mathrm{m}$ thick polyester sheet of Ref. [16], the 100-nm thick polystyrene sheets of Ref. [36], and the lipid monolayers of Refs. [32, 33], have $\zeta \sim 10^{-6}, 10^{-6}$, and $10^{-7}$, respectively. In these systems it would be extremely difficult to measure the compressibility effects derived here. On the other hand, there are softer sheets where these effects may not be as minute. For example, the Young modulus of common hydrogels is as low as $1-10 \mathrm{kPa}$. A $100-\mu \mathrm{m}$-thick layer of such a gel will have $\zeta$ of a few percent. The validity condition (46) for this system requires that $L>1 \mathrm{~cm}$. In fact, elastic moduli of very soft materials are notoriously hard to measure using conventional methods. The wrinkle wavelength is used to measure the bending modulus of sheets, from which, if the thickness is known, the Young modulus of the material can be indirectly inferred. One may be able to resolve the delicate compressibility effects in soft sheets, e.g., by high-precision measurement of the wrinkle wavelength. The wavelength derived above should be corrected for the fact that, in practice, one observes the compressed, rather than relaxed, lengths. Using Eqs. (21), we get the apparent wavelength as

$$
\lambda_{\text {apparent }}=\gamma_{\mathrm{w}} \lambda_{\mathrm{c}}=\lambda\left(\sqrt{1+\zeta^{2}}-\zeta\right)^{1 / 2} \simeq \lambda(1-\zeta / 2),
$$

where $\lambda=2 \pi[B /(\rho g)]^{1 / 4}$ is the commonly used wrinkle wavelength of an incompressible sheet. Thus, unlike the predicted wavelength in the relaxed frame, the apparent one will be slightly shorter than its incompressible counterpart. The correction will scale as $t^{5 / 4}$, on top of the dominant $t^{3 / 4}$ term. Such measurements may provide another, independent and more direct, handle for extracting small elastic moduli. 


\section{ACKNOWLEDGMENTS}

This work has been supported by the Israel Science Foundation (Grant No. 164/14).

[1] K. Y. C. Lee, Annu. Rev. Phys. Chem., 2008, 59, 771-791.

[2] L. D. Landau and E. M. Lifshitz, Theory of elasticity, 3rd edition, Butterworth-Heinemann, Oxford, 1998.

[3] J. M. T. Thompson and G. W. Hunt, A General Theory of Elastic Stability, Wiley, London, 1973.

[4] S. T. Milner, J.-F. Joanny and P. Pincus, Buckling of Langmuir monolayers. Europhys. Lett., 1989, 9, 495-500.

[5] E. Cerda and L. Mahadevan, Geometry and physics of wrinkling. Phys. Rev. Lett.,2003, 90, 074302.

[6] D. Vella, P. Aussillous and L. Mahadevan, Europhys. Lett., 2004, 68, 212.

[7] Q. Zhang and T. A. Witten, Phys. Rev. E, 2007, 76, 041608.

[8] J. Huang, M. Juszkiewicz, W. H. de Jeu, E. Cerda, T. Emrick, N. Menon and T. P. Russell, Science, $2007, \mathbf{3 1 7}, 650$.

[9] J. Huang, B. Davidovitch, C. D. Santangelo, T. P. Russell and N. Menon, Phys. Rev. Lett., 2010, $105,038302$.

[10] F. Brau, H. Vandeparre, A. Sabbah, C. Poulard, A. Boudaoud and P. Damman, Multiple-length-scale elastic instability mimics parametric resonance of nonlinear oscillators. Nature Phys. 7, 56-60 (2011)

[11] H. Vandeparre, M. Pineirua, F. Brau, B. Roman, J. Bico, C. Gay, W. Bao, C. N. Lau, P. M. Reis and P. Damman, Phys. Rev. Lett. 106, 224301 (2011).

[12] B. Davidovitch, R. D. Schroll and E. Cerda, Phys. Rev. E, 2012, 85, 066115.

[13] B. Li, Y.-P. Cao, X.-Q. Feng and H. Gao, Soft Matter, 2012, 8, 5728.

[14] G. W. Hunt, M. K. Wadee and N. Shiacolas, J. Appl. Mech., 1993, 60, 1033.

[15] S. H. Lee and A. M. Waas, Int. J. Non-Linear Mech., 1996, 31, 313.

[16] L. Pocivavsek, R. Dellsy, A. Kern, S. Johnson, B. Lin, K. Y. C. Lee and E. Cerda, Science 320, $2008,912$.

[17] P. M. Reis, F. Corson, A. Boudaoud and B. Roman, Phys. Rev. Lett., 2009, 103, 045501.

[18] B. D. Leahy, L. Pocivarsek, M. Meron, K. L. Lam, D. Salas, P. J. Viccaro, K. Y. C. Lee and B. Lin, Phys. Rev. Lett., 2010, 105, 058301.

[19] D. P. Holmes and A. J. Crosby, Phys. Rev. Lett., 2010, 105, 038303.

[20] H. Diamant and T. A. Witten, 2010, arXiv:1009.2487

[21] B. Audoly, Phys. Rev. E, 2011, 84, 011605.

[22] H. Diamant and T. A. Witten, Phys. Rev. Lett., 2011, 107, 164302.

[23] F. Brau, P. Damman, H. Diamant and T. A. Witten, Soft Matter, 2013, 9, 8177.

[24] H. Diamant and T. A. Witten, Phys. Rev. E, 2013, 88, 012401.

[25] M. Rivetti, Comptes Rendus Mecanique, 2013, 341, 333.

[26] M. R. Semler, J. M. Harris, A. B. Croll and E. K. Hobbie, Phys. Rev. E 88, 032409 (2013).

[27] V. Démery, B. Davidovitch and C. D. Santangelo, Phys. Rev. E, 2014, 90, 042401.

[28] M. Rivetti and S. Neukirch, J. Mech. Phys. Solids, 2014, 69, 143.

[29] O. Oshri, F. Brau and H. Diamant, Phys. Rev. E, 2015, 91, 052408.

[30] R. D. Schroll, E. Katifori and B. Davidovitch, Phys. Rev. Lett., 2011, 106, 074301.

[31] Y. Zhang and T. M. Fischer, J. Phys. Chem. B, 2005, 109, 3442.

[32] A. Gopal, V. Belyi, H. Diamant, T. A. Witten and K. Y. C. Lee, J. Phys. Chem. B, 2006, 110, 10220.

[33] A. Gopal and K. Y. C. Lee, J. Phys. Chem. B, 2006, 110, 22079.

[34] D. Vella, M. Adda-Bedia and E. Cerda, Soft Matter, 2010, 6, 5778.

[35] T. J. Wagner and D. Vella, Phys. Rev. Lett., 2011, 107, 044301.

[36] H. King, R. D. Schroll, B. Davidovitch and N. Menon, Proc. Natl. Acad. Sci. USA, 2012, 109, 9716.

[37] R. D. Schroll, M. Adda-Bedia, E. Cerda, J. Huang, N. Menon, T. P. Russell, K. B. Toga, D. Vella and B. Davidovitch, Phys. Rev. Lett., 2013, 111, 014301.

[38] M. Piñeirua, N. Tanaka, B. Roman and J. Bico, Soft Matter, 2013, 9, 10985.

[39] Handbook of Mathematical Functions, ed. M. Abramowitz and I. A. Stegun, Dover Publications, New York, 1972.

[40] A. Magnusson, M. Ristinmaa and C. Ljung, Int. J. Solids Struct., 2001, 38, 8441.

[41] O. Oshri and H. Diamant, Soft Matter, 2016, 12, 664.

[42] See detailed derivation in the Supplementary Material. 